\title{
VEGF ameliorates tubulointerstitial fibrosis in unilateral ureteral obstruction mice via inhibition of epithelial-mesenchymal transition
}

\author{
Yao-guo LIAN ${ }^{1}$, Qiu-gen ZHOU ${ }^{2}$, Ying-juan ZHANG ${ }^{1}$, Fa-lei ZHENG ${ }^{1, ~ * ~}$ \\ ${ }^{1}$ Division of Nephrology, Peking Union Medical College Hospital, Peking Union Medical College and Chinese Academy of Medical Sci- \\ ences, Beijing 100730, China; ${ }^{2}$ Division of Nephrology, Southern Medical University Nanfang Hospital, Guangzhou 510515, China
}

\begin{abstract}
Aim: Vascular endothelial growth factor (VEGF) has been shown to be a survival factor for renal tubular epithelial cells. In the present study, we investigated whether administration of VEGF ameliorates tubulointerstitial fibrosis in a mouse model of unilateral ureteral obstruction (UUO).

Methods: Thirty-six male CD-1 mice were randomly divided into three groups: sham-operation, UUO and UUO+VEGF group. VEGF (50 $\mu \mathrm{g} / \mathrm{kg}$ ) was subcutaneously injected twice daily from $\mathrm{d} 1$ to $\mathrm{d} 14$. Mice in each group were killed at $\mathrm{d} 3$, 7, or 14 after the operation, and the tubulointerstitial fibrosis was histopathologically evaluated. Human proximal tubular epithelial cells (HK-2) were used for in vitro study. The expression levels of $\alpha$-SMA, E-cadherin, TGF- $\beta 1$, CTGF, and BMP-7 in the kidney were determined using Western blot and RT-PCR.

Results: In the UUO mice, the degree of interstitial fibrosis was dramatically increased in a time-dependent manner. At $d$ 3, 7, and 14, both the mRNA and protein expression levels for $\alpha$-SMA, TGF- $\beta 1$, and CTGF were significantly upregulated, whereas those for E-cadherin and BMP-7 were significantly downregulated. At $d 3$ and 7, VEGF treatment significantly reduced interstitial fibrosis and the expression levels for $\alpha$-SMA, TGF- $\beta 1$, and CTGF, while significantly increased the expression of E-cadherin and BMP-7, as compared with the UUO mice. At d 14 after operation, no significant differences were observed in the expression of the examined markers between VEGFtreated mice and UUO mice, with the exception of CTGF. In HK-2 cells, VEGF blocked TGF- $\beta 1$-induced $\alpha$-SMA and vimentin expression and restored E-cadherin expression in a dose-dependent manner.

Conclusion: VEGF may ameliorate renal tubulointerstitial fibrosis at the early stage in UUO mice. This effect may be related to inhibition of VEGF on renal tubular epithelial-mesenchymal transition (EMT).
\end{abstract}

Keywords: VEGF; unilateral ureteral obstruction; renal tubulointerstitial fibrosis; epithelial-mesenchymal transition; transforming growth factor- $\beta 1$

Acta Pharmacologica Sinica (2011) 32: 1513-1521; doi: 10.1038/aps.2011.111; published online 10 Oct 2011

\section{Introduction}

Tubulointerstitial fibrosis is closely correlated with the progression of chronic renal disease and is a common final pathway leading to end-stage renal failure. Emerging evidence indicates that renal tubular epithelial-mesenchymal transition (EMT) is an important event in renal interstitial fibrosis ${ }^{[1]}$. All types of renal injury are potentially able to induce renal tubular cells to undergo EMT, generating mesenchymal cells, as demonstrated by experimental models, in vitro experiments, and renal biopsy studies ${ }^{[2]}$. EMT is the process in which renal tubular epithelial cells lose their epithelial phenotype followed

\footnotetext{
* To whom correspondence should be addressed. E-mail zhengfl46@vip.sina.com

Received 2011-03-23 Accepted 2011-07-11
}

by their acquisition of mesenchymal cell characteristics, contributing to continuous tubulointerstitial fibrosis ${ }^{[3]}$. Previous studies have demonstrated that transforming growth factor (TGF)- $\beta 1$ is an inducer of renal tubular epithelial cell EMT, whereas bone morphogenetic protein-7 (BMP-7) counteracts TGF- $\beta 1$-induced EMT and reverses chronic renal injury ${ }^{[4,5]}$. These data imply that there are several factors modulating the phenotype of renal tubular epithelial cells and that imbalance of these factors might lead to their change from the epithelial phenotype.

Vascular endothelial growth factor (VEGF) is an endothelialspecific growth factor that promotes endothelial cell proliferation, differentiation and survival, mediates endotheliumdependent vasodilatation, induces microvascular hyperpermeability and participates in interstitial matrix remodeling. In 
the kidney, VEGF expression is most prominent in glomerular podocytes and tubular epithelial cells ${ }^{[6,7]}$. Several studies have shown that VEGF may relieve experimental renal diseases, such as anti-Thy1 glomerulonephritis, thrombotic microangiopathy and anti-glomerular basement membrane glomerulonephritis $^{[8-10]}$. Moreover, reduced expression of VEGF has been observed in tubulointerstitial fibrosis ${ }^{[11-13]}$.

VEGF treatment in a rat model of remnant kidneys inhibited loss of peritubular capillaries and improved renal function and peritubular capillary endothelial cell proliferation ${ }^{[14]}$. Furthermore, VEGF has been shown to induce vasculogenesis and tubulogenesis ${ }^{[15,16]}$. In addition, VEGF has been shown to induce a proliferative response and to prevent hydrogen peroxide-induced apoptosis and necrosis in renal tubular epithelial cells ${ }^{[17]}$.

We previously reported that pentoxifylline treatment inhibited tubulointerstitial fibrosis in a rat model of obstructive nephropathy and prevented loss of $\mathrm{VEGF}^{[18]}$. In addition, our previous in vitro studies indicated that reduced expression of VEGF might contribute to tubular EMT ${ }^{[19]}$.

The above-mentioned studies suggest that VEGF may be a renoprotective factor important for maintenance of the epithelial phenotype of renal tubular cells. In the present study, we used a mouse model of obstructive nephropathy to test whether VEGF could ameliorate tubulointerstitial fibrosis and EMT and whether it could affect the expression of TGF- $\beta 1$, connective tissue growth factor (CTGF) and BMP-7.

\section{Materials and methods Reagents}

VEGF $_{121}$ was purchased from R\&D Systems (Minneapolis, MN, USA). Blood urea nitrogen and serum creatinine were detected using a kit from Sigma (St Louis, MO, USA). Antia-smooth muscle actin (a-SMA) was obtained from Sigma (St Louis, MO, USA); anti-E-cadherin, anti-TGF- $\beta 1$, anti-CTGF, anti-BMP-7, anti-vimentin and an anti-actin polyclonal antibody and ECL were obtained from Santa Cruz Biotechnology (Santa Cruz, CA, USA). The Histostain ${ }^{\mathrm{TM}}$-plus kit was from Zymed Laboratories (South San Francisco, CA, USA). The TRIzol reagent was from Life Technologies/BRL (Rockville, MD, USA). M-MLV reverse transcriptase and Taq polymerase were purchased from Promega (Madison, WI, USA). BCA protein assay kits were purchased from Beyotime (Haimen, China).

\section{Experimental design}

Thirty-six male CD-1 mice (21-24 g, Grade III, Peking Union Medical College Hospital) were divided into two 1:2 matched groups. The sham group (group A) was subjected to surgical manipulation without ureteral ligation; the unilateral ureteral obstruction (UUO) group was subjected to left ureteral ligation. The animals were housed in individual cages under conditions of constant temperature and humidity on a 12-h light/dark cycle. Food and water were provided ad libitum. Protocols for animal experimentation and maintenance were approved by the Animal Ethics Committee at our institute and carried out in accordance with institutional guidelines. For the surgery, anesthesia with sodium pentobarbital (40 $\mathrm{mg} / \mathrm{kg}$, ip) was provided, and the left ureter was ligated with silk sutures at two points and was cut between the ligatures. The UUO mice were randomly divided into two groups: one group (group C) received $\mathrm{VEGF}_{121}(50 \mu \mathrm{g} / \mathrm{kg}$, subcutaneous $(\mathrm{sc})$, twice daily, $n=12$ ) from d 1 to $\mathrm{d} 14$ after ligation ${ }^{[14]}$, and the other group (group B) received vehicle (normal saline, sc, $n=12$ ) as a control. The $\mathrm{VEGF}_{121}$ isoform was chosen because it is the only isoform of VEGF that has no heparin-binding ability and, therefore, results in therapeutically effective plasma levels when administered subcutaneously. Four mice from each group (including group $\mathrm{A}$, the sham-operated mice, to serve as negative controls) were killed on d 3 after operation, four mice on $d 7$, and four mice on $d 14$. The kidneys were decapsulated and divided into several parts. One part was fixed in $10 \%$ neutral buffered formalin and processed for histological analysis. Another part was dissected to isolate the cortex, which was quickly frozen in liquid nitrogen and stored at $-70^{\circ} \mathrm{C}$ for protein and total RNA extraction. Blood was collected for serum creatinine and blood urea nitrogen measurements according to the protocol of the commercial kit.

\section{Histologic examination}

Kidneys were removed, fixed in $10 \%$ neutral formalin, embedded in paraffin, sectioned (2.0-3.0 $\mu \mathrm{m}$ thickness), and stained with Masson trichrome using routine procedures. Evaluation of tubulointerstitial fibrosis was performed. In each section, 20 randomly selected fields were examined under $\times 400$ magnification for assessment of the degree of tubulointerstitial fibrosis. The degree of tubulointerstitial fibrosis was scored in Masson-stained sections as the ratio of the positive stain area (blue) to that of the whole area $(n=20)$. Tubulointerstitial fibrosis was examined in each mouse and then averaged for the four mice from each group.

\section{Cell culture}

Human proximal tubular epithelial cells (HK-2) (from Dr Xiong-zhong RUAN, Royal Free and University College Medical School, UK) were cultured and maintained in Dulbecco's modified Eagle's medium/Ham's F-12 containing 10\% fetal bovine serum as previously described ${ }^{[18]}$. All studies were carried out in overnight serum-deprived cells at 90\%-100\% confluency.

\section{Reverse transcription-PCR}

Total RNA was isolated from the cortex of the ligated kidneys or from cultured cells using the TRIzol reagent according to the manufacturer's instructions. First-strand cDNA was made from total RNA using the M-MLV transcriptase. PCR was performed in a DNA Thermal Cycler with the primers listed in Table 1. Primers were designed with OLIGO software (version 6) and synthesized by the Shanghai Sangon Biological Engineering Company (Beijing, China).

Reactions were incubated at $95^{\circ} \mathrm{C}$ for $5 \mathrm{~min}$, followed by the indicated number of cycles of $30 \mathrm{~s}$ at $94^{\circ} \mathrm{C}$ and $30 \mathrm{~s}$ at $56^{\circ} \mathrm{C}$ 
Table 1. Primers used in RT-PCR experiments.

\begin{tabular}{|c|c|c|c|}
\hline Gene & Primers & Sequences & $\begin{array}{l}\text { Length of } \\
\text { products }\end{array}$ \\
\hline \multicolumn{4}{|c|}{ Subunit of mouse } \\
\hline \multirow[t]{2}{*}{ GAPDH } & Forward & 5'-GCCTTCCGTGTTCCTACCC-3' & 166 bp \\
\hline & Reverse & 5'-TGAAGTCGCAGGAGACAACC-3' & \\
\hline \multirow[t]{2}{*}{$\alpha-S M A$} & Forward & 5'-CCGAGCGTGAGATTGTCC-3' & 358 bp \\
\hline & Reverse & 5'-CTTCATGGTGCTGGGTGC-3' & \\
\hline \multirow[t]{2}{*}{ E-cadherin } & Forward & 5'-CAAAGTGGCGACAGACGG-3' & 483 bp \\
\hline & Reverse & 5'-GACACGGCATGAGAATAGAGG-3' & \\
\hline \multirow[t]{2}{*}{ TGF- $\beta 1$} & Forward & 5'-TGGTGGACCGCAACAACG-3' & 394 bp \\
\hline & Reverse & 5'-CGACGTTTGGG GCTGATC-3' & \\
\hline \multirow[t]{2}{*}{ CTGF } & Forward & 5'-CTATCCCACCAAAGTGAGAACG-3' & 417 bp \\
\hline & Reverse & 5'-TTGCCACAA GCTGTCCAGTC-3' & \\
\hline \multirow[t]{2}{*}{ BMP-7 } & Forward & 5'-AGGGAGTCGGACCTCTTCTTG-3' & 472 bp \\
\hline & Reverse & 5'-CCTCACAGTAGTAGGCAGCATAGC-3' & \\
\hline \multicolumn{4}{|c|}{ Subunit of human } \\
\hline \multirow[t]{2}{*}{ GAPDH } & Forward & 5'-CCACCCATGGCAAATTCCATGGCA-3' & 323 bp \\
\hline & Reverse & 5'-TGCTAAGCAGTTGTTGGTGCAGGA-3' & \\
\hline \multirow[t]{2}{*}{$\alpha-S M A$} & Forward & 5'-GCTCACGGAG GCACCCCTGAA-3' & 590 bp \\
\hline & Reverse & 5'-CTGATAGGACATTGTTAGCAT-3' & \\
\hline \multirow[t]{2}{*}{ E-cadherin } & Forward & 5'-СТСАСАТТТСССААСТСС-3' & 405 bp \\
\hline & Reverse & 5'-ACCTTCCATGACAGACCC-3' & \\
\hline \multirow[t]{2}{*}{ Vimentin } & Forward & 5'-GGCTCAGATTCAGGAACAGC-3' & 327 bp \\
\hline & Reverse & 5'-GCTTCAACGGCAAA GTTCTC-3' & \\
\hline
\end{tabular}

for a-SMA, E-cadherin, vimentin, TGF- $\beta 1, \mathrm{CTGF}$, and BMP-7 $\left(58^{\circ} \mathrm{C}\right.$ for $\left.\mathrm{GAPDH}\right), 30 \mathrm{~s}$ at $72^{\circ} \mathrm{C}$, and a final extension at $72{ }^{\circ} \mathrm{C}$ for $10 \mathrm{~min}$. There were 35 cycles of PCR for a-SMA, E-cadherin, vimentin, TGF- $\beta 1, \mathrm{CTGF}$, and BMP-7, and 28 cycles for GAPDH. The PCR products were electrophoresed using $2 \%$ agarose and their signal intensity was determined with the UVI soft UVIband Windows application V97.04. The resulting signal intensities were normalized to that of GAPDH.

\section{Western blot analysis}

Kidney tissues or cultured cells were homogenized in RIPA lysis buffer (1\% NP40, 0.1\% SDS, $100 \mu \mathrm{g} / \mathrm{mL}$ phenylmethylsulfonyl fluoride, $0.5 \%$ sodium deoxycholate, in PBS) on ice. The supernatants were collected after centrifugation at $12000 \times \mathrm{g}$ at $4^{\circ} \mathrm{C}$ for $20 \mathrm{~min}$. Protein concentration was determined using a BCA protein assay kit, and whole lysates were mixed with an equal amount of $2 \times$ SDS loading buffer $(125 \mathrm{mmol} / \mathrm{L}$ Tris- $\mathrm{HCl}$, $4 \%$ SDS, $20 \%$ glycerol, $100 \mathrm{mmol} / \mathrm{L} \mathrm{DTT}$, and $0.2 \%$ bromophenol blue). Samples were heated at $100^{\circ} \mathrm{C}$ for $5 \mathrm{~min}$ and were separated on SDS-polyacrylamide gels. The separated proteins were then transferred to a PVDF membrane. The membrane blots were first probed with a primary antibody. After incubation with horseradish peroxidase-conjugated second antibody, autoradiograms were prepared using the enhanced chemiluminescent system to visualize the protein antigen. The signals were recorded using X-ray film, and the signal intensities were determined using the UVI soft UVI band Windows application
V97.04. The primary antibodies that we used included antia-SMA (1:300), anti-E-cadherin (1:400), anti-vimentin (1:200), anti-TGFß1 (1:200), anti-CTGF (1:400), anti-BMP-7 (1:600), and anti-actin (1:2000). The results were normalized to the signal intensity of $\beta$-actin, which was used as an internal control.

\section{Confocal microscopy}

HK-2 cells treated with TGF- $\beta 1$ (5 $\mu \mathrm{g} / \mathrm{L})$ alone, co-treated with TGF- $\beta 1(5 \mu \mathrm{g} / \mathrm{L})$ and different concentrations of VEGF $(0.1,1,10$, and $100 \mu \mathrm{g} / \mathrm{L})$ or left untreated were cultured in eight-chamber glass slides for $48 \mathrm{~h}$. Cells were fixed with $4 \%$ paraformaldehyde in phosphate-buffered saline (PBS) (20 min; room temperature), permeabilized with $0.1 \%$ Triton $\mathrm{X}-100$ in PBS (15 min), and incubated in normal goat serum (30 min), followed by application of a monoclonal anti-a-SMA antibody (1:50 in BSA/PBS, Sigma) and a polyclonal anti-E-cadherin antibody (1:100 in BSA/PBS, Santa Cruz Biotechnology) (overnight, $\left.4^{\circ} \mathrm{C}\right)$. The slides were then washed with PBS, incubated in rhodamine (TRITC)-conjugated goat anti-mouse IgG (1:50 in BSA/PBS; Proteintech Group, Chicago, IL, USA) and fluorescein (FITC)-conjugated affinipure goat anti-rabbit IgG (1:50 in BSA/PBS; Proteintech Group, Chicago, IL, USA) (60 min; room temperature), washed with PBS, and incubated in 4',6-diamidino-2-phenylindole (DAPI) for $3 \mathrm{~min}$, followed by a washing with PBS. Microscopy was performed with a Leica TCS SP2 AOBS confocal microscope (Leica Micro-Systems, Heidelberg, Germany) using an excitation wavelength of 488 $\mathrm{nm}$ from an Argon laser and an emission spectra between 520 and $570 \mathrm{~nm}$. Collection parameters remained constant for all samples.

\section{Statistical analyses}

All data were expressed as the mean \pm SD. Statistical analyses were carried out using SPSS 11.5 software for Windows. Statistical significance was evaluated by one-way ANOVA, and the statistical significance between two groups was evaluated by $t$-test. Values of $P<0.05$ were considered statistically significant.

\section{Results}

\section{VEGF improves histopathologic changes in UUO mice}

After ureteral ligation, mice developed hydronephrosis with progressive dilation of the renal pelvis and thinning of the renal cortex. Serum creatinine and blood urea nitrogen levels remained unchanged at all time points (data not shown). From d 3 to 14, renal tubular damage after UUO increased progressively and was characterized by dilation with flattened epithelium at d 3, atrophy and thickness of the tubular basement membrane at $\mathrm{d} 7$ and destruction of tubules at $\mathrm{d} 14$. In parallel, a progressive expansion of the interstitial space and fibrosis gradually developed, as revealed by Masson trichrome staining (Figure 1). There was no difference in tubulointerstitial fibrosis between the UUO group and the VEGF-treated group at d $14(P>0.05)$. Meanwhile, the tubulointerstitial fibrosis score was reduced by approximately $8 \%$ at $\mathrm{d} 3$ and $18 \%$ at d 7 in the VEGF-treated group compared with $15 \% \pm 1 \%$ at $\mathrm{d} 3$ 


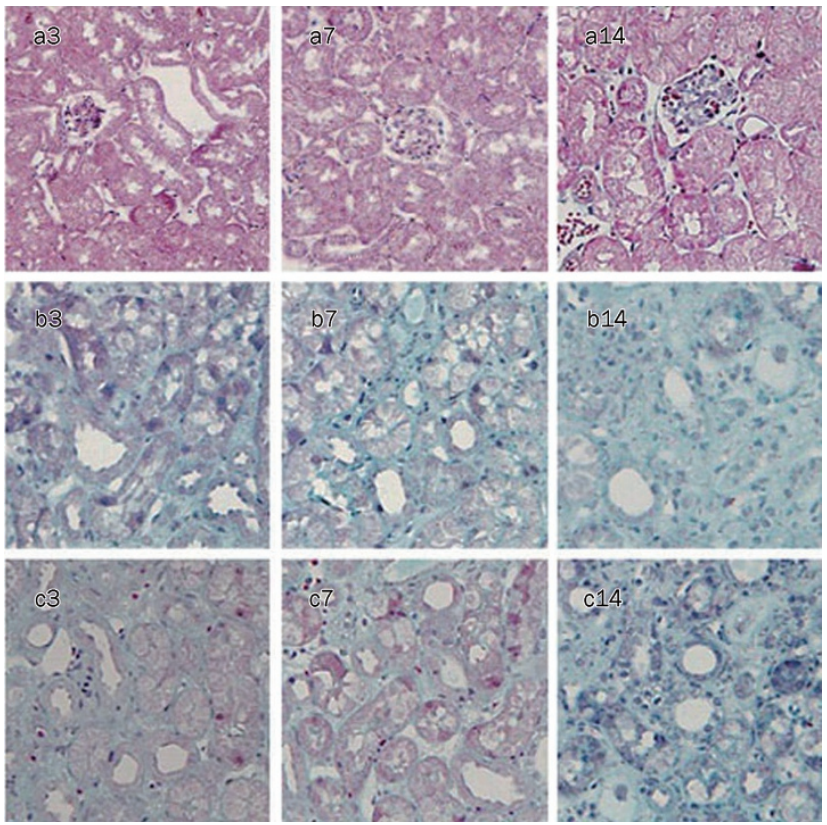

Figure 1. Representative histopathology in sham mice (a), UUO mice (b) and VEGF-treated mice (c) at d 3, 7, and 14, respectively (Masson stain, $\times 400)$. D 3 after ligation, few fibrosis appeared in interstitial space. D 7 and 14 after ligation, moderate and severe fibrosis took place, respectively.

and $36 \% \pm 3 \%$ at $d 7$ in the UUO group $(P<0.05$, Figure 2$)$.

VEGF inhibits $\alpha$-SMA expression and restores E-cadherin expression in UUO mice

EMT is an important event in renal interstitial fibrosis. During the process of EMT, epithelial cells lose their polygonal morphology and adhesive cell contacts (E-cadherin) and acquire fibroblast-like characteristics, including an elongated shape, expression of mesenchymal markers (eg, a-SMA and vimentin)

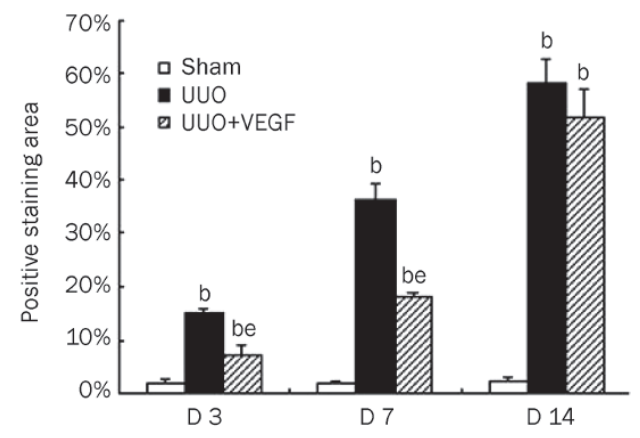

Figure 2. Semiquantitative tubulointerstitial fibrosis score in sham mice, UUO mice and VEGF-treated mice at $d 3,7$, and 14 , respectively. The fibrosis score was markedly increased in UUO mice compared with sham mice. Compared with UUO mice, the fibrosis score was significantly reduced in VEGF treated mice at $d 3$ and 7 . The values were shown as pecentage of the staining area to selected area and expressed as mean \pm SD of 3 independent experiments. ${ }^{b} P<0.05$ vs sham group; ${ }^{\mathrm{e}} P<0.05$ vs UUO group. and increased motility ${ }^{[20-22]}$. To investigate the effect of VEGF on EMT in the kidney, a-SMA and E-cadherin protein and mRNA expression levels were determined by Western blot and RT-PCR, respectively, in UUO mice treated with or without VEGF. As shown in Figure 3 and 4, in the UUO group, a-SMA expression was significantly upregulated, whereas E-cadherin expression was significantly downregulated compared with the sham group from $\mathrm{d} 3$ to $14(P<0.05)$. VEGF treatment reduced a-SMA expression and improved E-cadherin expression at $\mathrm{d} 3$ and 7 compared with the UUO group $(P<0.05)$. No differences in the expression of a-SMA or E-cadherin were observed between the VEGF-treated and UUO mice at $\mathrm{d} 14(P>0.05)$.

A
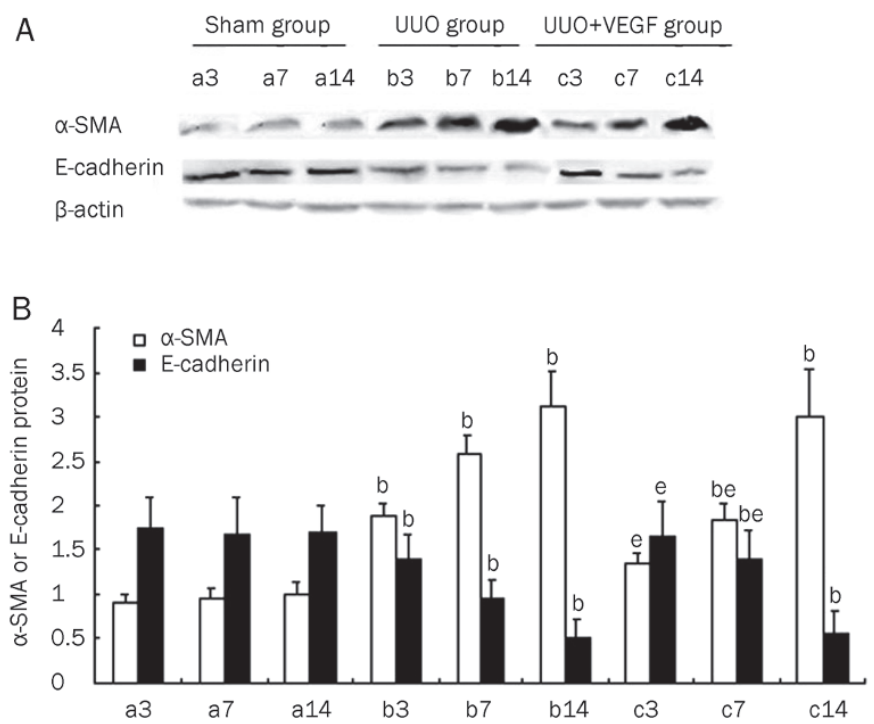

Figure 3. The expressions of $\alpha$-SMA and E-cadherin protein in sham mice (a), UUO mice (b) and VEGF-treated mice (c) at d 3, 7, and 14, respectively. (A) Representative Western blot of $\alpha$-SMA and E-cadherin in sham and UUO mice treated with or without VEGF. (B) Compared with UUO group, VEGF treatment significantly inhibited $\alpha$-SMA expression and increased E-cadherin expression at $d 3$ and 7. Results were shown as ratio of optical density for $\alpha$-SMA or E-cadherin to that of $\beta$-actin and presented as mean \pm SD of 3 independent experiments. ${ }^{b} P<0.05$ vs respective sham mice; ${ }^{e} P<0.05$ vs respective UUO mice.

VEGF affects the expression of TGF- $\beta 1$, CTGF, and BMP-7 in UUO mice

Several cytokines, including TGF- $\beta 1$, CTGF, and BMP-7, are thought to be involved in the pathogenesis of EMT. To examine whether VEGF could modulate the expression of these cytokines, the kidney protein and mRNA levels of TGF- $\beta 1$, CTGF and BMP-7 were measured in UUO mice treated with or without VEGF. Compared with the sham group, the expression levels of TGF- $\beta 1$ and CTGF in the UUO group were significantly upregulated, whereas the expression of BMP-7 was significantly downregulated from d 3 to $14(P<0.05)$. Importantly, VEGF treatment in the UUO model resulted 
A Sham group UUO group UUO+VEGF group $\begin{array}{llllllllll}\text { a3 } & \text { a7 } & \text { a14 } & \text { b3 } & \text { b7 } & \text { b14 } & \text { c3 } & \text { c7 } & \text { c14 }\end{array}$

$\alpha-S M A$

E-cadherin

GAPDH
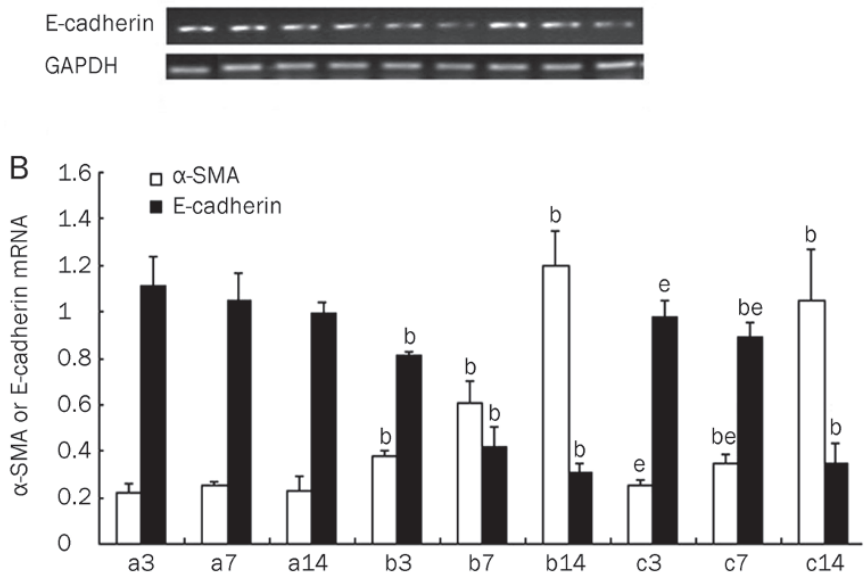

Figure 4. The expressions of $\alpha$-SMA and E-cadherin mRNA in sham mice (a), UUO mice (b) and VEGF-treated mice (c) at d 3, 7, and 14, respectively. (A) Representative RT-PCR of $\alpha$-SMA and E-cadherin in sham and UUO mice treated with or without VEGF. (B) Compared with UUO group, VEGF treatment inhibited $\alpha$-SMA expression and increased $E$-cadherin expression at $d 3$ and 7 . Results were shown as ratio of optical density for $\alpha-S M A$ or E-cadherin to that of GAPDH and presented as mean \pm SD of 3 independent experiments. ${ }^{b} P<0.05$ vs respective sham mice; ${ }^{e} P<0.05$ vs respective UUO mice.

in reduced expression of TGF- $\beta 1$ and CTGF expression and increased BMP-7 expression at $\mathrm{d} 3$ and 7 compared with the untreated UUO group $(P<0.05)$. No differences in the expression of TGF- $\beta 1$ and BMP-7 were observed between the VEGFtreated and untreated UUO mice at d $14(P>0.05)$. However, VEGF treatment did result in reduced CTGF expression at d 14 $(P<0.05)$. Thus, VEGF treatment prevented the production of TGF- $\beta 1$ and loss of BMP-7 expression at both the mRNA and protein levels at $\mathrm{d} 3$ and $\mathrm{d} 7$, and VEGF treatment inhibited the production of CTGF at $\mathrm{d} 3,7$, and $14(P<0.05)$ (Figures 5 and 6).

\section{VEGF inhibits EMT induced by TGF- $\beta 1$ in cultured HK-2 cells}

Confocal microscopy study demonstrated that E-cadherin, a phenotypic marker of epithelial cells, was distributed in the cytoplasm, in the nucleolus and at the cell-cell junctions, whereas a-SMA, a phenotypic marker of mesenchymal cells, was not detected under normal conditions (Figure 7A). The addition of TGF- $\beta 1(5 \mu \mathrm{g} / \mathrm{L})$ significantly induced $\alpha$-SMA expression, but inhibited E-cadherin expression (Figure 7B). VEGF $(0.1-100 \mu \mathrm{g} / \mathrm{L})$ treatment restored E-cadherin expression and intercellular junctions and dramatically abrogated TGF- 31 -induced a-SMA expression in a dose-dependent manner (Figure 7C).

To further confirm that VEGF blocked TGF- $\beta 1$-induced EMT in HK-2 cells, we measured the expression of a-SMA, vimentin and E-cadherin in HK-2 cells treated with TGF- $\beta 1$ $(5 \mu \mathrm{g} / \mathrm{L})$ in the presence or absence of VEGF $(0.1-100 \mu \mathrm{g} / \mathrm{L})$.
A

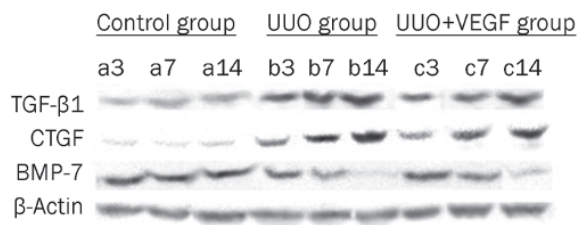

$\mathrm{B}$

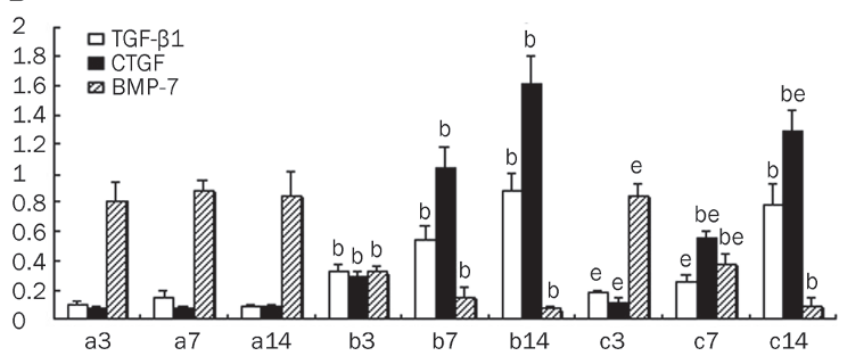

Figure 5. The protein levels of TGF- $\beta 1$, CTGF and BMP-7 in sham mice (a), UUO mice (b) and VEGF-treated mice (c) at d 3, 7, and 14, respectively. (A) Representative western blot of TGF- $\beta 1$, CTGF and BMP-7 in sham and UUO mice treated with or without VEGF. (B) Compared with UUO group, VEGF treatment significantly inhibited the expressions of TGF- $\beta 1$ and CTGF and increased BMP-7 expression. Results were shown as ratio of optical density for TGF- $\beta 1$, CTGF, or BMP-7 to that of $\beta$-actin and presented as mean \pm SD of 3 independent experiments. ${ }^{b} P<0.05$ vs respective sham mice; ${ }^{\mathrm{e}} \mathrm{P}<0.05$ vs respective UUO mice.

A

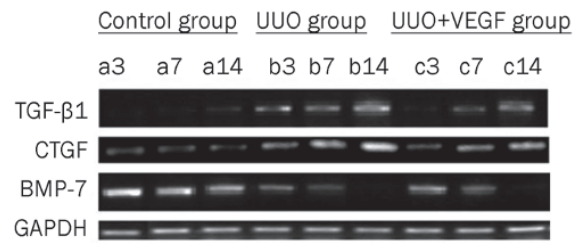

B

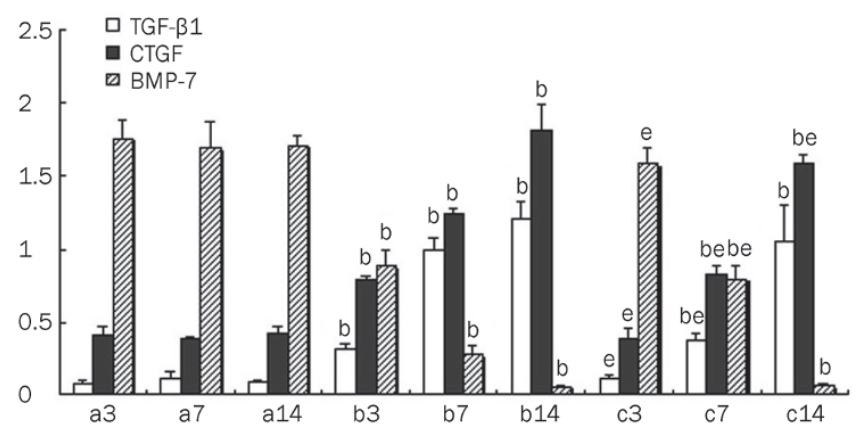

Figure 6. The mRNA levels of TGF- $\beta 1$, CTGF and BMP-7 in sham mice (a), UUO mice (b) and VEGF-treated mice (c) at d 3, 7, and 14, respectively. (A) Representative RT-PCR of TGF- $\beta 1$, CTGF, and BMP-7 in sham and UUO mice treated with or without VEGF. (B) Compared with UUO group, VEGF treatment significantly blocked the expressions of TGF- $\beta 1$ and CTGF and improved expression of BMP-7. Results were shown as ratio of optical density for TGF- $\beta 1$, CTGF, or BMP-7 to that of GAPDH and presented as mean \pm SD of 3 independent experiments. ${ }^{b} P<0.05$ vs respective sham mice; ${ }^{\mathrm{e}} P<0.05$ vs respective UUO mice. 

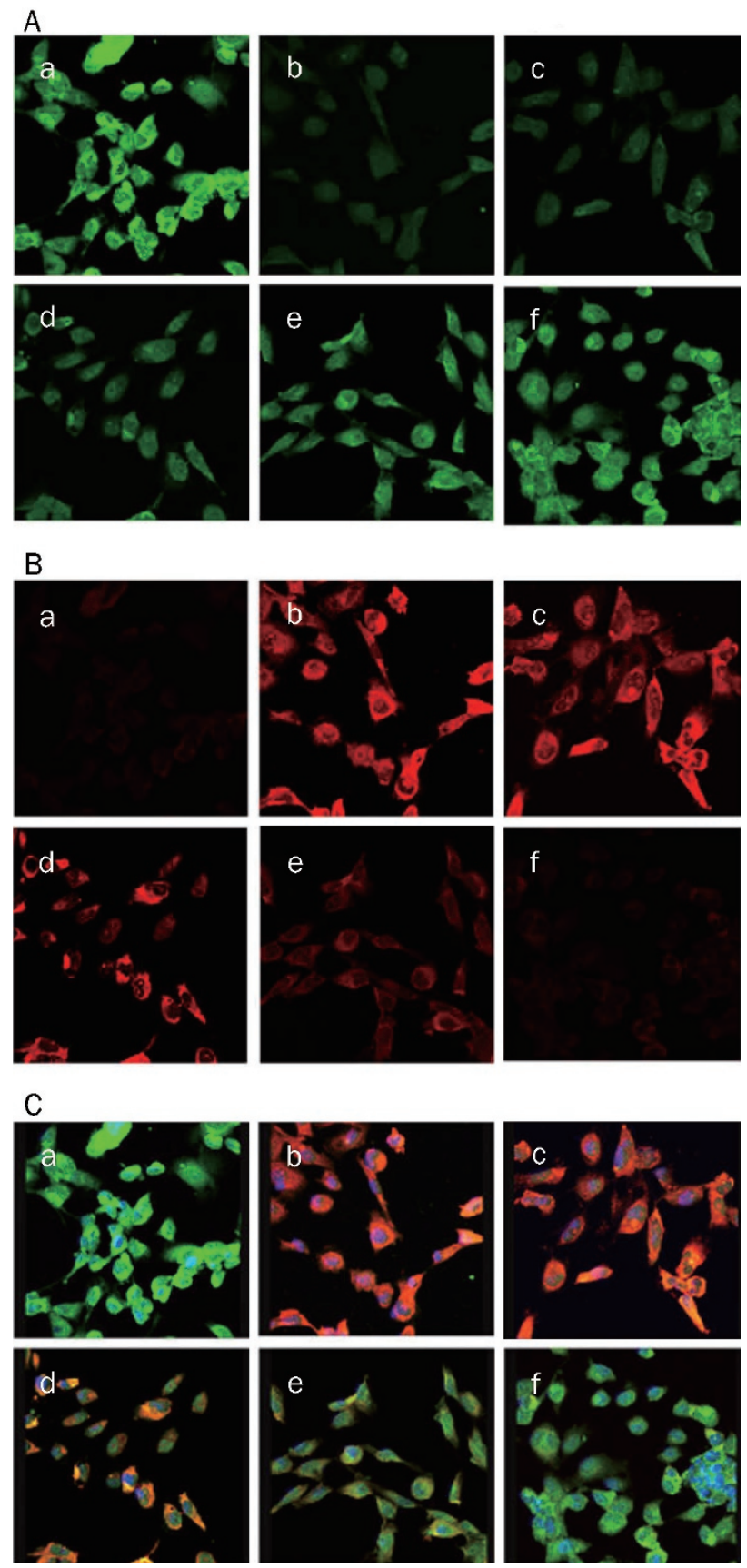

Figure 7. Expressions of E-cadherin and $\alpha$-SMA in HK-2 cells treated with $5 \mu \mathrm{g} / \mathrm{L}$ TGF- $\beta 1$ in the presence or absence of VEGF at $0.1-100$ $\mu \mathrm{g} / \mathrm{L}$, evaluated by confocal microscopy. The expression of E-cadherin (A) and $\alpha$-SMA (B) were observed by confocal microscopic image. The colocalization (C) of $\alpha$-SMA and E-cadherin was stained yellow. a, no treatment; b, $5 \mu \mathrm{g} / \mathrm{L}$ TGF- $\beta 1 ; \mathrm{c}, 5 \mu \mathrm{g} / \mathrm{L}$ TGF- $\beta 1$ and $0.1 \mu \mathrm{g} / \mathrm{L}$ VEGF; $d, 5$ $\mu \mathrm{g} / \mathrm{L}$ TGF- $\beta 1$ and $1 \mu \mathrm{g} / \mathrm{L}$ VEGF; e, $5 \mu \mathrm{g} / \mathrm{L}$ TGF- $\beta 1$ and $10 \mu \mathrm{g} / \mathrm{L}$ VEGF; f, 5 $\mu \mathrm{g} / \mathrm{L}$ TGF- $\beta 1$ and $100 \mu \mathrm{g} / \mathrm{L}$ VEGF. $\times 100$.

TGF- $\beta 1$ alone induced expression of a-SMA and vimentin and inhibited expression of E-cadherin at both the protein and mRNA levels. In contrast, co-treatment with VEGF dramatically abrogated the TGF- $\beta 1$-induced increases in protein and
mRNA expression of a-SMA and vimentin and also restored the protein and mRNA expression of E-cadherin in HK-2 cells in a dose-dependent manner. Moreover, at concentrations of $100 \mu \mathrm{g} / \mathrm{L}$, VEGF almost completely blocked the TGF- $\beta 1$ induced changes in a-SMA, vimentin and E-cadherin protein and mRNA expression $(P<0.05)$ (Figures 8 and 9 ).
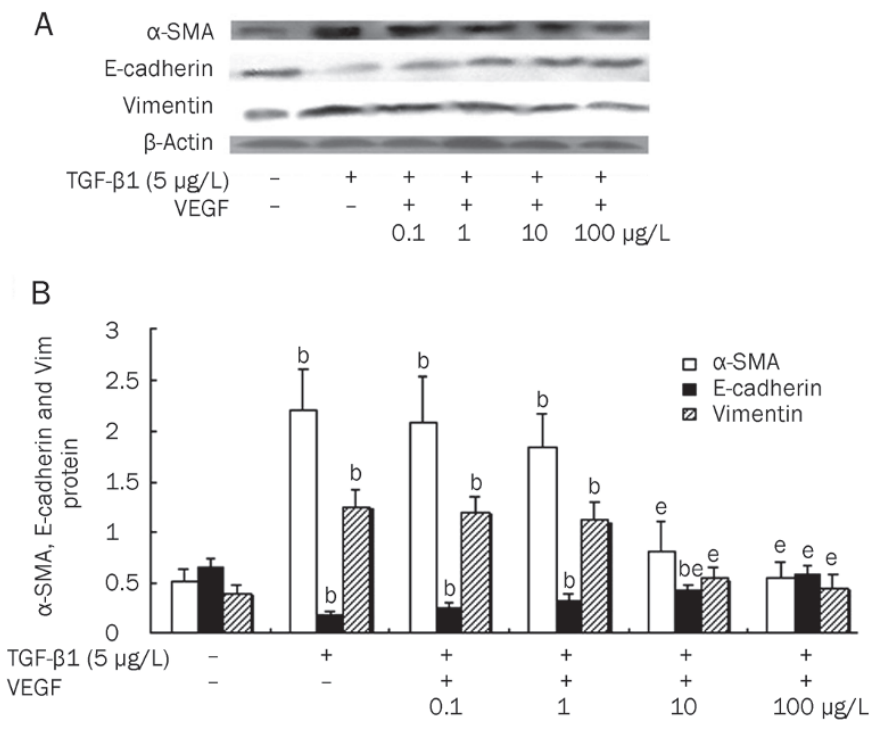

Figure 8. Protein levels of $\alpha$-SMA, E-cadherin and vimentin in HK-2 cell treated with $5 \mu \mathrm{g} / \mathrm{L}$ TGF- $\beta 1$ alone, co-treated with $5 \mu \mathrm{g} / \mathrm{L}$ TGF- $\beta 1$ and VEGF at $0.1-100 \mu \mathrm{g} / \mathrm{L}$, or untreated for $48 \mathrm{~h}$. (A) Representative Western blot of $\alpha$-SMA, E-cadherin and vimentin in HK-2 cells. (B) Compared with HK-2 cells treated with TGF- $\beta 1$ alone, VEGF treatment significantly inhibited the expressions of $\alpha$-SMA and vimentin and improved the expression of E-cadherin in a dose-dependent manner. Results were shown as ratio of optical density for $\alpha$-SMA, E-cadherin, or vimentin to that of $\beta$-actin and presented as mean \pm SD of 3 independent experiments. ${ }^{b} P<0.05$ vs respective HK2 cells untreated; ${ }^{e} P<0.05$ vs respective HK2 cells treated with $5 \mu \mathrm{g} / \mathrm{L}$ TGF- $\beta 1$ alone

\section{Discussion}

Renal interstitial fibrosis is a common pathological process leading to end-stage renal disease ${ }^{[23]}$. The UUO model has been widely used as a model of tubulointerstitial fibrosis ${ }^{[24]}$. The UUO model is characterized by renal myofibroblast activation, tubular atrophy and interstitial fibrosis with minimal glomerular lesions in which TGF- $\beta 1$ is considered to play an important role ${ }^{[24,25]}$. In our study, the obstructed kidneys displayed marked tubulointerstitial injury starting on d 3 after surgery, but glomerular cells remained nearly untouched.

In the present study, the effect of VEGF on tubulointerstitial fibrosis and EMT was tested. Our results from in vivo experiments showed that VEGF inhibited tubulointerstitial fibrosis and EMT. The potential mechanisms of VEGF action include inhibition of TGF- $\beta 1$ and CTGF expression in target cells and the improvement of BMP-7 expression. In addition, we showed that TGF- $\beta 1$ induced EMT and that VEGF directly 
A

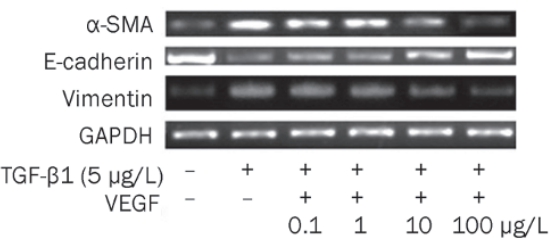

B

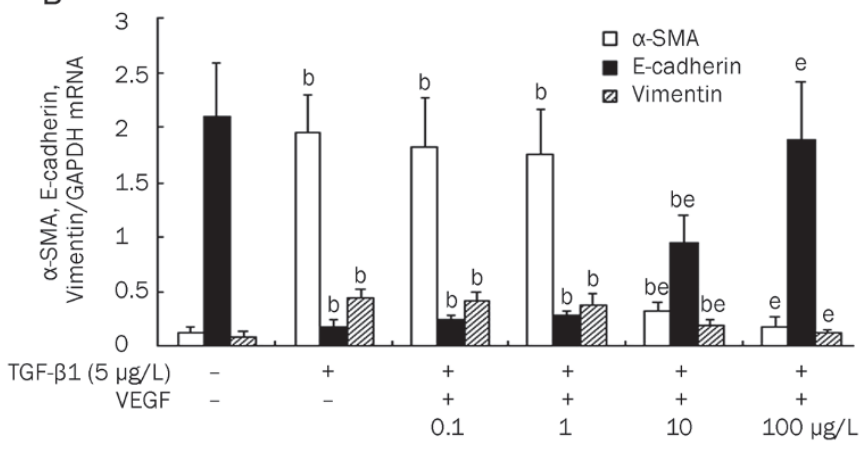

Figure 9. The mRNA levels of $\alpha$-SMA, E-cadherin and vimentin in HK-2 cell treated with $5 \mu \mathrm{g} / \mathrm{L}$ TGF- $\beta 1$ in the presence or absence of VEGF at $0.1-100 \mu \mathrm{g} / \mathrm{L}$ for $48 \mathrm{~h}$. (A) Representative RT-PCR of $\alpha$-SMA, E-cadherin, and vimentin in HK-2 cells. (B) Compared with HK-2 cells treated with TGF- $\beta 1$ alone, VEGF treatment significantly inhibited the expressions of $\alpha$-SMA and vimentin and improved the expression of E-cadherin in a dose-dependent manner. Results were shown as ratio of optical density for $\alpha$-SMA, E-cadherin, or vimentin to that of GAPDH and presented as mean \pm SD of 3 independent experiments. ${ }^{b} P<0.05$ vs respective HK2 cells untreated; ${ }^{e} P<0.05$ vs respective HK2 cells treated with $5 \mu \mathrm{g} / \mathrm{L}$ TGF- $\beta 1$ alone.

inhibited TGF- $\beta 1$-induced EMT in cultured human renal tubular epithelial cells in vitro. Thus, for the first time to our knowledge, we provide evidence that the inhibitory effect of VEGF on tubulointerstitial fibrosis is likely associated with an amelioration of the EMT process.

It is well known that EMT is a novel mechanism involved in renal interstitial fibrosis ${ }^{[26,27]}$. In obstructive nephropathy induced by UUO, the tubular epithelial cells lost expression of the epithelial cell marker E-cadherin and acquired mesenchymal features, such as expression of a-SMA and vimentin and production of interstitial matrix components, indicating that they were at a transitional stage between epithelium and mesenchyme ${ }^{[28-30]}$. In the UUO model, we observed an early induction of a-SMA at $\mathrm{d} 3$ followed by a more robust a-SMA induction at $\mathrm{d} 7$ after surgery. The induction of a-SMA was closely associated with loss of the expression of the epithelial marker E-cadherin. This result is consistent with previous studies $^{[31,32]}$. These data suggest that VEGF attenuates a-SMA expression and improves E-cadherin expression on $\mathrm{d} 3$ and 7 after surgery, but fails to function at d 14, indicating that VEGF inhibits EMT during the early stage of injury in the UUO mouse model.

EMT is regulated by many factors and in different ways, with TGF- $\beta 1$ being the most potent inducer that is capable of initiating and completing the entire EMT course and with
BMP-7 acting as an EMT inhibitor both in vitro and in vivo ${ }^{[33-37]}$. CTGF can promote EMT both directly and as a downstream mediator of TGF- $\beta 1^{[38]}$. In our study, VEGF treatment reduced the expression of TGF- $\beta 1$ and CTGF and upregulated the expression of BMP-7. In accordance with the central role of TGF- $\beta 1$ and its downstream signaling cascades in the activation of the cellular mechanisms that underlie the progression of EMT ${ }^{[39]}$, VEGF-mediated inhibition of TGF- $\beta 1$ and CTGF expression would abolish much of their profibrotic effects. Our results demonstrate that VEGF blocked fibrosis and EMT likely through its influence on TGF- $\beta 1$, CTGF and BMP-7 expression in UUO mice.

VEGF is a survival factor for vascular endothelial cells and plays an important role in the homeostasis of the vascular endothelium in addition to being a survival factor for renal tubular epithelial cells that is involved in renal tubule construction ${ }^{[40-42]}$. VEGF expression in human and mouse kidney is confined to podocytes and tubular epithelial cells, and renal tubular epithelial cells are an important cellular resource of VEGF in the kidney. There may be intrinsic renoprotective factors that maintain the epithelial phenotype of tubular cells in vivo, and loss of these factors may contribute to EMT. VEGF may be a candidate for this type of factor because reduced expression of VEGF has been observed in tubulointerstitial fibrosis $^{[11-13]}$. Furthermore, it has been reported that depolymerized hyaluronan could inhibit developmental EMT of mouse endocardial cushions by inducing $\mathrm{VEGF}^{[43]}$ and that supplementation with VEGF improved cyclosporine-mediated interstitial injury ${ }^{[4]}$. Therefore, modulation of VEGF expression might be a therapeutic approach for the prevention of progressive renal disease.

In summary, our data provide further in vivo and in vitro evidence that VEGF may improve tubulointerstitial fibrosis by inhibiting EMT at the early stage of injury. Our findings may have clinical implications for the use of VEGF in patients with early chronic tubulointerstitial injury. It remains to be investigated whether increased dosage of VEGF would be more effective in preventing EMT at later stages of injury.

\section{Acknowledgements}

This project was supported by the National Natural Science Foundation of China (№ 30570854).

\section{Author contribution}

Fa-lei ZHENG designed the research; Yao-guo LIAN performed the research; Fa-lei ZHENG, Yao-guo LIAN, and Yingjuan ZHANG contributed new analytical tools and reagents; Yao-guo LIAN analyzed the data; and Yao-guo LIAN and Qiu-gen ZHOU wrote the paper.

\section{References}

1 Kalluri R, Neilson EG. Epithelial-mesenchymal transition and its implications for fibrosis. J Clin Invest 2003; 112: 1776-84.

2 Rastaldi MP. Epithelial-mesenchymal transition and its implications for the development of renal tubulointerstitial fibrosis. J Nephrol 2006; 19: 407-12. 
3 Lee JM, Dedhar S, Kalluri R, Thompson EW. The epithelial-mesenchymal transition: new insights in signaling, development, and disease. J Cell Biol 2006; 172: 973-81.

4 Zeisberg M, Hanai J, Sugimoto H, Mammoto T, Charytan D, Strutz F, et al. BMP-7 counteracts TGF-beta1-induced epithelial-to-mesenchymal transition and reverses chronic renal injury. Nat Med 2003; 9: 9648.

5 Xu Y, Wan J, Jiang D, Wu X. BMP-7 counteracts TGF-beta1-induced epithelial-to mesenchymal transition in human renal proximal tubular epithelial cells. J Nephrol 2009; 22: 403-10.

6 Schrijvers BF, Flyvbjerg A, De Vriese AS. The role of vascular endothelial growth factor (VEGF) in renal pathophysiology. Kidney Int 2004; 65: 2003-17.

7 Ferrara N. Role of vascular endothelial growth factor in the regulation of angiogenesis. Kidney Int 1999; 56: 794-814.

8 Wada Y, Morioka T, Oyanagi-Tanaka Y, Yao J, Suzuki Y, Gejyo F, et al. Impairment of vascular regeneration precedes progressive glomerulosclerosis in anti-Thy1 glomerulonephritis. Kidney Int 2002; 61: 432-43.

9 Kim YG, Suga SI, Kang DH, Jefferson JA, Mazzali M, Gordon KL, et al. Vascular endothelial growth factor accelerates renal recovery in experimental thrombotic microangiopathy. Kidney Int 2000; 58: 2390-9.

10 Shimizu A, Masuda Y, Mori T, Kitamura H, Ishizaki M, Sugisaki Y, et al. Vascular endothelial growth factor165 resolves glomerular inflammation and accelerates glomerular capillary repair in rat antiglomerular basement membrane glomerulonephritis. J Am Soc Nephrol 2004; 15: 2655-65.

11 Song YR, You SJ, Lee YM, Chin HJ, Chae DW, Oh YK, et al. Activation of hypoxia-inducible factor attenuates renal injury in rat remnant kidney. Nephrol Dial Transplant 2010; 25: 77-85.

12 Sun D, Feng J, Dai C, Sun L, Jin T, Ma J, et al. Role of peritubular capillary loss and hypoxia in progressive tubulointerstitial fibrosis in a rat model of aristolochic acid nephropathy. Am J Nephrol 2006; 26 : 363-71.

13 Burt LE, Forbes MS, Thornhill BA, Kiley SC, Chevalier RL. Renal vascular endothelial growth factor in neonatal obstructive nephropathy. I. Endogenous VEGF. Am J Physiol Renal Physiol 2007; 292: F158-67.

14 Kang DH, Hughes J, Mazzali M, Schreiner GF, Johnson RJ. Impaired angiogenesis in the remnant kidney model: II. Vascular endothelial growth factor administration reduces renal fibrosis and stabilizes renal function. J Am Soc Nephrol 2001; 12: 1448-57.

15 Turo A, Norwood VF, Carey RM, Gomez RA. Vascular endothelial growth factor induces nephrogenesis and vasculogenesis. J Am Soc Nephrol 1999; 10: 2125-34.

16 Villegas G, Lange-Sperandio B, Tufro A. Autocrine and paracrine functions of vascular endothelial growth factor (VEGF) in renal tubular epithelial cells. Kidney Int 2005; 67: 449-57.

17 Kanellis J, Fraser S, Katerelos M, Power DA. Vascular endothelial growth factor is a survival factor for renal tubular epithelial cells. Am J Physiol Renal Physiol 2000; 278: F905-15.

18 Zhou QG, Zheng FL, Hou FF. Inhibition of tubulointerstitial fibrosis by pentoxifylline is associated with improvement of vascular endothelial growth factor expression. Acta Pharmacol Sin 2009; 30: 98-106.

19 Zhou QG, Zheng FL, Wen YB, Tan XY, Duan L, Li Y. Altered expression of vascular endothelial growth factor and its receptors in transdifferentiated human proximal tubular epithelial cells induced by transforming growth factor beta1. Zhongguo Yi Xue Ke Xue Yuan Xue Bao 2005; 27: 325-31.

20 Bakin AV, Tomlinson AK, Bhowmick NA, Moses HL, Arteaga CL.
Phosphatidylinositol 3-kinase function is required for transforming growth factor $\beta$-mediated epithelial to mesenchymal transition and cell migration. J Biol Chem 2000; 275: 36803-10.

21 Fan JM, Ng YY, Hill PA, Nikolic-Paterson DJ, Mu W, Atkins RC, et al. Transforming growth factor- $\beta$ regulates tubular epithelial-myofibroblast transdifferentiation in vitro. Kidney Int 1999; 56: 1455-67.

22 Bozic M, de Rooij J, Parisi E, Ortega MR, Fernandez E, Valdivielso JM. Glutamatergic signaling maintains the epithelial phenotype of proximal tubular cells. J Am Soc Nephrol 2011; 22: 1099-111.

23 Isaka Y, Takahara S, Imai E. Chronic deteriorating renal function and renal fibrosis. Contrib Nephrol 2008; 159: 109-21.

24 Chevalier RL. Molecular and cellular pathophysiology of obstructive nephropathy. Pediatr Nephrol 1999; 13: 612-9.

25 Klahr S, Morrissey J. Obstructive nephropathy and renal fibrosis. Am J Physiol Renal Physiol 2002; 283: F861-75.

26 Liu Y. New insights into epithelial-mesenchymal transition in kidney fibrosis. J Am Soc Nephrol 2010; 21: 212-22.

$27 \mathrm{Liu} \mathrm{YH}$. Epithelial to mesenchymal transition in renal fibrogenesis: pathologic significance, molecular mechanism, and therapeutic intervention. J Am Soc Nephrol 2004; 15: 1-12.

28 Yang J, Liu Y. Dissection of key events in tubular epithelial to myofibroblast transition and its implications in renal interstitial fibrosis. Am J Pathol 2001; 159: 1465-75.

29 Yang J, Liu Y. Blockage of tubular epithelial to myofibroblast transition by hepatocyte growth factor prevents renal interstitial fibrosis. J Am Soc Nephrol 2002; 13: 96-107.

30 Iwano M, Plieth D, Danoff TM, Xue C, Okada H, Neilson EG. Evidence that fibroblasts derive from epithelium during tissue fibrosis. J Clin Invest 2002; 110: 341-50.

31 Yang J, Dai C, Liu Y. Hepatocyte growth factor gene therapy and angiotensin II blockade synergistically attenuate renal interstitial fibrosis in mice. J Am Soc Nephrol 2002; 13: 2464-77.

32 Yang J, Shultz RW, Mars WM, Wegner RE, Li Y, Dai C, et al. Disruption of tissue-type plasminogen activator gene in mice reduces renal interstitial fibrosis in obstructive nephropathy. J Clin Invest 2002; 110: 1525-38.

33 Bottinger EP, Bitzer M. TGF- $\beta$ signaling in renal disease. J Am Soc Nephrol 2002; 13: 2600-10.

34 Chaudhuri V, Zhou L, Karasek M. Inflammatory cytokines induce the transformation of human dermal microvascular endothelial cells into myofibroblasts: a potential role in skin fibrogenesis. J Cutan Pathol 2007; 34: 146-53.

35 Wahab NA, Mason RM. A critical look at growth factors and epithelialto-mesenchymal transition in the adult kidney. Nephron Exp Nephrol 2006; 104: e129-34.

36 Morrissey J, Hruska K, Guo G, Wang S, Chen Q, Klahr S. Bone morphogenetic protein-7 improves renal fibrosis and accelerates the return of renal function. J Am Soc Nephrol 2002; 13: S14-21.

37 Nguyen TQ, Goldschmeding R. Bone morphogenetic protein-7 and connective tissue growth factor: novel targets for treatment of renal fibrosis? Pharm Res 2008; 25: 2416-26.

38 Zhang C, Meng X, Zhu Z, Liu J, Deng A. Connective tissue growth factor regulates the key events in tubular epithelial to myofibroblast transition in vitro. Cell Biol Int 2004; 28: 863-73.

39 Wendt MK, Allington TM, Schiemann WP. Mechanisms of the epithelial-mesenchymal transition by TGF-beta. Future Oncol 2009; 5: 1145-68.

40 Karihaloo A, Karumanchi SA, Cantley WL, Venkatesha S, Cantley LG, Kale S. Vascular endothelial growth factor induces branching morphogenesis/tubulogenesis in renal epithelial cells in a neuropilindependent fashion. Mol Cell Biol 2005; 25: 7441-8. 
41 Kang DH, Joly AH, Oh SW, Hugo C, Kerjaschki D, Gordon KL, et al. Impaired angiogenesis in the remnant kidney model: I. Potential role of vascular endothelial growth factor and thrombospondin-1. J Am Soc Nephrol 2001; 12: 1434-47.

42 Katavetin P, Miyata T, Inagi R, Tanaka T, Sassa R, Ingelfinger JR, et al. High glucose blunts vascular endothelial growth factor response to hypoxia via the oxidative stress-regulated hypoxia-inducible factor/ hypoxia-responsible element pathway. J Am Soc Nephrol 2006; 17: 1405-13.
43 Rodgers LS, Lalani S, Hardy KM, Xiang X, Broka D, Antin PB, et al. Depolymerized hyaluronan induces vascular endothelial growth factor, a negative regulator of developmental epithelial-to-mesenchymal transformation. Circ Res 2006; 99: 583-9.

44 Kang DH, Kim YG, Andoh TF, Gordon KL, Suga S, Mazzali M, et al. Post-cyclosporine-mediated hypertension and nephropathy: amelioration by vascular endothelial growth factor. Am J Physiol Renal Physiol 2001; 280: F727-36. 